\title{
The strength prediction of concrete frozen in the initial through the grey theory
}

\author{
G.Y. Wang ${ }^{1, a}$, S. Xie ${ }^{1, a^{*}}$, Z. Cheng ${ }^{1, a}$ \\ ${ }^{1}$ Shenyang jian zhu University,Shenyang,Liaoning, China \\ a474030570@qq.com
}

Keywords: the initial cold,the intensity change, the gray theory, the residuals

Abstract.Revealed the strength variation of concrete C30 and C50 after the cold in the initial .Using the gray prediction theory which from the cybernetics to analyze the test results after the initial cold.The results show that the results obtained by the formula is consistent with the experimental results, the residuals are within the control range. The experimental in cold for 120 days is much closer than theoretical values when compared with the residual for 90 days.Research shows that with the increase of initial freezing time, the loss rate of concrete strengthincreased.And the residuals showed a significant increasing trend when the number of freeze-thaw cycles increased.

\section{Introduction}

At present, the freeze-thaw cycle [1] is one of the main factors on durability of concrete. Concrete frost can generally be divided into three types, The first is the new pouring concretedirectly affected by the cold before initial setting of the concrete. The second is shortage cold for concrete, which did not reach the curing age of 28 days, also known as the initial cold. The third is be cold after reaching the age of 28 days. The main research in this article is the second types of cold. Since the reform and opening up, the number of freezethaw cycles of a large number of concrete structure or component has reached about 30 times, while most of our test number is a multiple of 25 times. It's very difficult for usto get the variation of concrete strength in the condition of freezethaw cycles about 30 times. A large number of test for the variation of concrete strength is just to analyze the phenomenon, but not for qualitative analysis, much less the theoretical analysis of the qualitative. Therefore, for the above problems, the author mainly uses the gray system theory GM $(1,1)$ model, respectively havingthe variation in intensity of concrete C30 and C50 modeled, calculated and analyzed.

\section{Experiment overview}

The author used literature data in [2]. The test uses standard concrete test block for $150 \mathrm{~mm}$ $\times 150 \mathrm{~mm} \times 150 \mathrm{~mm}$, the grade of concrete strength are C30 and C50. Fastfreeze method is used in the test. Firstly soaked the test block into water with the temperature $(20 \pm 2){ }^{\circ} \mathrm{C}$ for 4 days after curing for 7 days, then take the freezethaw test. In each freezethaw cycle, the test block were frozen for 12 hours then melted at standard temperature for 12 hours, that is to say a freezethaw cycle for one day. The freezethaw cycle time arerespectively 90 days, 100 days, 110 days and 120 days. The experimental groups are shown in Table 1.

Table 1 The experimental group

\begin{tabular}{cccccc}
\hline & \multicolumn{5}{c}{ Freezing time/d } \\
\cline { 2 - 6 } $\begin{array}{c}\text { Concrete } \\
\text { strength/MPa }\end{array}$ & 0 & 90 & 100 & 110 & 120 \\
\hline C30 & Y1-1 & Y1-2 & Y1-3 & Y1-4 & Y1-5 \\
C50 & Y2-1 & Y2-2 & Y2-3 & Y2-4 & Y2-5 \\
\hline
\end{tabular}




\section{Computational model}

GM (Grey Model) model, namely the gray prediction model. GM $(1,1)$ is a forecasting model which represents a firstorder variables of differential equation model. According to the gray system theory and the Hua xi [3] theory the gray prediction model was obtained. The prediction model using freezethaw cycles $\mathrm{N}$ for the variable, which is a derivative of high dispersion number $\mathrm{X}^{(0)}=\left\{\mathrm{X}^{(0)}\left(\mathrm{N}_{\mathrm{t}}\right) \quad \mid \mathrm{N}_{\mathrm{t}} \in \mathrm{R}^{+}, \mathrm{i}=1,2,3 \ldots, \mathrm{n}\right\}$, and set up the gray model $\mathrm{GM}(1,1)$ model.

First, assume a discrete sequence

$$
\mathrm{X}\left(0=\left(\mathrm{x}^{(0)}{ }_{(1)}, \mathrm{x}^{(0)}{ }_{(2), \ldots .} \mathrm{x}^{(0)}{ }_{(\mathrm{n})}\right)\right.
$$

According to the parameters derived the capacity areas for

$$
a \in\left(\frac{-2}{n+1}, \frac{2}{n+1}\right) ; \quad \sigma_{(k)} \in\left(e^{\frac{-2}{n+1}}, e^{\frac{2}{n+1}}\right)
$$

In the formula: $\sigma_{(k)}$ represents sequence level.

According to the discrete sequence of the test result, the ratio test was taken in advance.

$$
\sigma_{(N)}=\frac{X_{(N-1)}}{X_{(N)}} \in\left(e^{-\frac{2}{n+1}}, e^{\frac{2}{n+1}}\right)
$$

If the the original data does not meet the requirements of level ratio test, must have it transformed to another until it meets the conditions.In order to obtain high accuracy $\operatorname{GM}(1,1)$ model, the sequen ce level should be as close to 1 ratio after the treatment, namely: as small as possible. The author mai nly adopts the transform method for translation. Let $\mathrm{q}$ be the given constant, $\mathrm{x}$ is the original sequenc $\mathrm{e}$, translation of $\mathrm{y}$ for $\mathrm{x}$ seri-es $x=\left(x_{(1)}, x_{(2)}, \mathrm{L}, x_{(n)}\right), y=\left(y_{(1)}, y_{(2)}, \mathrm{L}, y_{(n)}\right)$ and

$y_{(k)}=x_{(k)}+Q_{(k)}, Q_{(k)}=$ const,$\forall k$ If $\varepsilon$ specified value for $\delta_{y(k)}$. Then

$$
\begin{aligned}
& Q_{(k)}=\frac{\Delta_{x(k)}}{\varepsilon}-x_{(k)} \\
& Q^{*}=\max _{k} Q_{(k)}=\max _{k}\left(\frac{\Delta_{x(k)}}{\varepsilon}-x_{(k)}\right)
\end{aligned}
$$

It can be seen that the mechanism of the shift is select $Q^{*}$ as the appropriate translation value, to make ensure the transformation sequences with ratio deviation.

If the level ratio test or after treatment with level ratio test can met, the gray differential equation with this data established.

$$
\frac{d X^{(0)}(N)}{d m}+a X^{(0)}=u
$$

According to the gray differential equation above, the basic solution basic can be obtained as follows:

$$
\begin{aligned}
& X_{(N)}^{(0)}=c e^{-a m}+b \\
& Y=\left[\begin{array}{llll}
X^{(0)}{ }_{\left(N_{1}\right)} & X^{(0)}{ }_{\left(N_{2}\right)} & \cdots & X^{(0)}{ }_{\left(N_{n}\right)}
\end{array}\right]^{T}
\end{aligned}
$$




$$
\begin{aligned}
& G^{T}=\left[\begin{array}{llll}
e^{-a N_{1}} & e^{-a N_{2}} & \cdots & e^{-a N_{n}} \\
1 & 1 & \cdots & 1
\end{array}\right] \\
& {\left[\begin{array}{ll}
c & b
\end{array}\right]^{T}=\left(\mathrm{G}^{\mathrm{T}} \mathrm{G}\right)^{-1} \mathrm{G}^{\mathrm{T}} \mathrm{Y}}
\end{aligned}
$$

\section{The initial cold comcrete strength predictive}

The strength formula were obtained by testfitting,but the numerically is less. In order to understan $\mathrm{d}$ the precision, we use gray theory to predict. theexperimental values of strength concrete after initial cold are shown in Table 2.

Table 2 The early concrete strength value after enduring the initial cold

\begin{tabular}{cccccc}
\hline & \multicolumn{5}{c}{ Freezing time/d } \\
\cline { 2 - 6 } Concrete strength /MPa & 0 & 90 & 100 & 110 & 120 \\
\cline { 2 - 6 } & 31.2 & 30.05 & 28.31 & 25.93 & 22.71 \\
C30 & 51.16 & 50.35 & 48.26 & 45.88 & 43.42 \\
\hline
\end{tabular}

\section{Prediction the strength of concrete $\mathbf{C 3 0}$}

Take level ratio test for the raw data to determine the feasibility of the model:

$$
\sigma_{(N)}=\frac{X_{(N-1)}}{X_{(N)}} \in\left(e^{-\frac{2}{n+1}}, e^{\frac{2}{n+1}}\right)
$$

According to the above formula to calculate whether the ratio $\sigma_{(N)}$ is in the admissible covering:

$$
\begin{aligned}
& \sigma_{(90)}=0.963 \notin(0.978,1.022), \quad \sigma_{(100)}=0.942 \notin(0.98,1.02), \\
& \sigma_{(110)}=0.916 \notin(0.982,1.018), \quad \sigma_{(120)}=0.876 \notin(0.984,1.017)
\end{aligned}
$$

That is $\sigma_{y N}=\left[\begin{array}{llll}0.963 & 0.942 & 0.916 & 0.876\end{array}\right]$

It can be seen that data conversion processi-ng must be carried out. Now select the translation for data processing. Then select formula $y_{(N)}=X_{(N)}+Q_{(N)}$, wherein $Q_{(N)}$ represents the translated nume rical value, to convert the calculation:

$$
\begin{aligned}
& \Delta_{x}(90)=\left|X_{90}-X_{0}\right| \\
& \Delta_{x}=\left|\begin{array}{llll}
1.15 & 1.74 & 2.38 & 3.22
\end{array}\right|
\end{aligned}
$$

In order to select the proper translation values, the selection level ratio deviation $\varepsilon$ should not greater than 0.01 , that is $\varepsilon \leq 0.01$.

$$
Q_{(N)}=\frac{\Delta_{x}(N)}{\varepsilon}-X_{(N)}
$$

According to the formula can be drawn:

$$
\begin{aligned}
& Q=\left[\begin{array}{llll}
84.95 & 145.69 & 212.07 & 299.29
\end{array}\right] \\
& Q^{*}=\max _{N} Q_{(N)}=299.29
\end{aligned}
$$

Therefore after translation the relationship is for: 


$$
\begin{aligned}
& y_{(N)}=X_{(N)}+299.29 \\
& y_{(N)}=\left[\begin{array}{lllll}
330.49 & 329.34 & 327.6 & 325.22 & 322
\end{array}\right] \\
& \sigma_{y N}=\left[\begin{array}{llll}
1.003 & 1.005 & 1.007 & 1.01
\end{array}\right] \\
& \delta_{y}=\left|1-\sigma_{y N}\right| \\
& \delta_{y}=\left[\begin{array}{llll}
0.003 & 0.005 & 0.007 & 0.01
\end{array}\right] \\
& \delta_{y}(\max )=\max _{N} \delta_{y}<0.01
\end{aligned}
$$

This shows that the average value of 299.29 can guarantee the transformation sequencY of the lev el ratio deviation $\varepsilon \leq 0.01$. Obviously the level ratio deviation falls within the range of allowed values after the transformation .

$$
\begin{aligned}
& B^{T}=\left[\begin{array}{cccc}
-329.915 & -328.47 & -326.41 & -323.61 \\
1 & 1 & 1 & 1
\end{array}\right] Y_{N}=\left[\begin{array}{llll}
-0.0128 & -0.174 & -0.238 & -0.322
\end{array}\right]^{T} \\
& {\left[\begin{array}{ll}
a & u
\end{array}\right]^{T}=\left(B^{T} B\right)^{-1} B^{T} Y_{N}=\left[\begin{array}{c}
-0.05224 \\
7101.5
\end{array}\right]} \\
& \Delta f_{c}^{*}=c e^{0.05224 N}+b \\
& Y=\left[\begin{array}{lllll}
330.49 & 329.34 & 327.6 & 325.22 & 322
\end{array}\right]^{T} \\
& G^{T}=\left[\begin{array}{ccccc}
1 & 110.12 & 185.68 & 313.06 & 527.84 \\
1 & 1 & 1 & 1 & 1
\end{array}\right] \\
& {\left[\begin{array}{cc}
c & b
\end{array}\right]^{T}=\left(G^{T} G\right)^{-1} G^{T} Y=\left[\begin{array}{c}
-0.0166 \\
330.68
\end{array}\right]} \\
& \Delta f_{c}^{*}=-0.0166 e^{0.05224 N}+330.68
\end{aligned}
$$

The resulting equation is

$$
\Delta f_{c}=-0.0166 e^{0.05224 N}+31.39
$$

Now the results of this formula with the experimental results were compared, the specific data in Table 2.Now introducing the concept of residuals to test the prediction equation.

$$
\varepsilon_{(k)}=\frac{\text { The actual values }- \text { The modle values }}{\text { The actual values }} \times 100 \%
$$

It can be seen from Table 2 that the residuals calculated are small,and are within the scope.For mula (8) can be used to forecast the changes of intensity in this experiment, and can make up for a gr eater scope. It can be seen from table 3 that the test value is lower than the theoretical value without the cold, but the test value is higher than the theoretical value in the cold.Compared with the residual of 90 days, the experimental values are closer than the theoretical values of 120 days .

Table 3 Test compared with calculated value

\begin{tabular}{cccc}
\hline Freezing time & The test value $/ \mathrm{MPa}$ & The calculate value/MPa & $\varepsilon / \%$ \\
\hline 0 & 31.2 & 31.37 & -0.54 \\
90 & 30.05 & 29.56 & 1.63 \\
120 & 22.71 & 22.63 & 0.35 \\
\hline
\end{tabular}


It can be seen from the above calculation and comparison that formula (8) can better predict the $\mathrm{s}$ trength value changes of concrete $\mathrm{C} 30$ varies with the time in the role of initialcold. The contrasted $\mathrm{c}$ urve of experimental and theoretical values is shown in Figure 1.

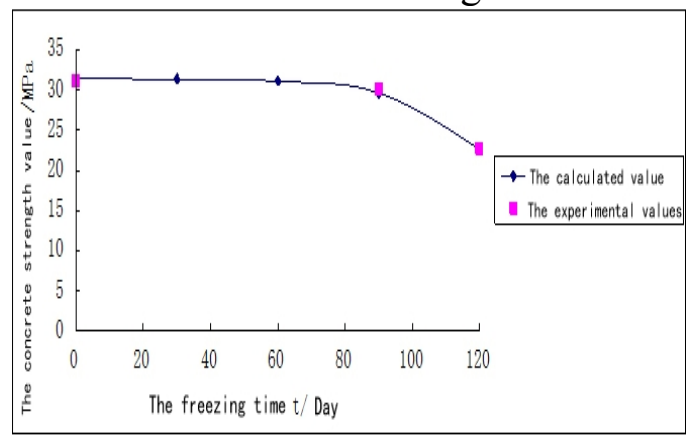

Fig. 1 Calculated value compared with the theoretical value

It can be seen from Figure 1: The experimental value and theoretical value trend consistent compl etely, and the calculated values coincide in case the test value. The overall trend of the curve is smoot $\mathrm{h}$ at the beginning, then it begins to reduce speed after 60 days and the curve down to further acceler ate the pace after 90 days.

\section{Prediction the strength of concrete C50}

Use the strength prediction method of concrete $\mathrm{C} 30$ to predict the strength of concrete $\mathrm{C} 50$, finally get the equation:

$\Delta f^{\prime}{ }_{c}=-0.0023 e^{0.068 N}+51.43$

Now take the residuals to test the feasibility of this formula.

Table 4 Test compared with calculated value

\begin{tabular}{cccc}
\hline Freezing time & The test value/MPa & The calculate value/MPa & $\varepsilon / \%$ \\
\hline 0 & 51.16 & 51.43 & -0.53 \\
90 & 50.35 & 50.38 & -0.06 \\
120 & 43.42 & 43.38 & 0.09 \\
\hline
\end{tabular}

The residual values calculated in Table 4 are small, and are included within the scope. From that perspective to derive that equation (10) can be used to predict the intensity changes in the test. It can be seen from table 2 that the test value is lower than the theoretical value without the cold, but the test value is higher than the theoretical value in the cold. Compared with the residual of 90 days, the experimental values are closer than the theoretical values of 120 days. Experimental and theoretical values contrast curve shown in Figure 2.

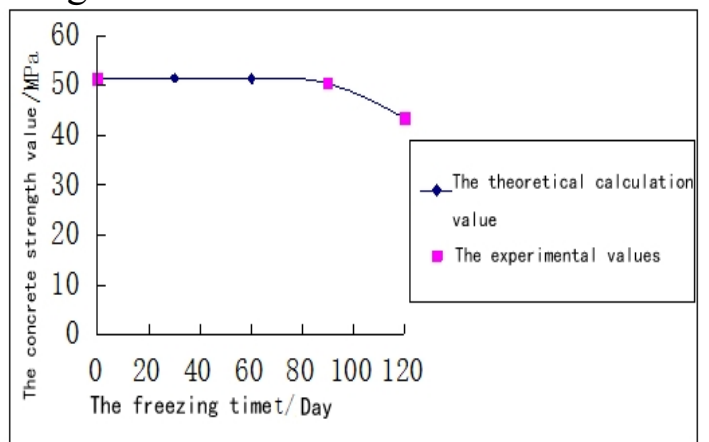

Fig. 2 Calculated value compared with the theoretical value

It can be seen from Figure 1: The experimental value and theoretical value trend consistent completely, and the calculated values coincide in case the test value. The overall trend of the curve is smooth at the beginning, then it begi-ns to reduce speed after 60 days and the curve down to further accelerate the pace after 90 days. 


\section{Comparison the strength prediction of concrete $\mathbf{C 3 0}$ and $\mathbf{C 5 0}$}

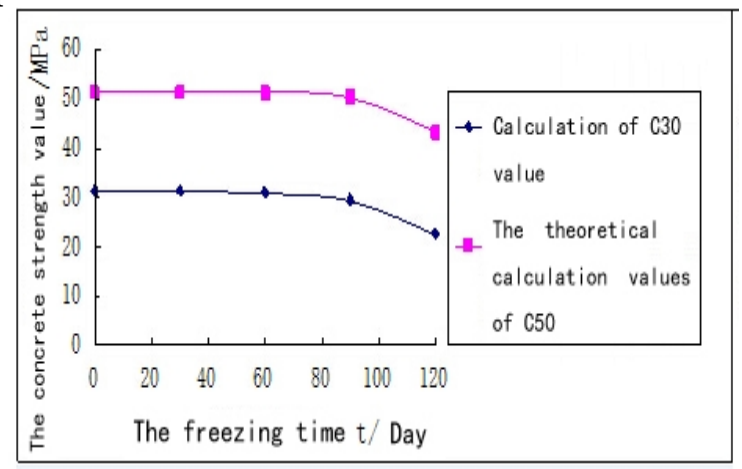

Fig. 2.3 C30 and C50 contrast

It can be seen from Figure 2.3 that the trend of the two curve is consistent when compared to C30 and $\mathrm{C} 50$. The overall trend of the curve is smooth at the beginning, then it begins to reduce speed after 60 days and the curve down to further accelerate the pace after 90 days. The rate of decline of ordinary concrete and high strength concrete are basically the same in the previous 30 days. But the speed of high strength concrete falling faster than ordinary concrete between 30 days to 60 days, then the rate of decline maintains such a contrast value.

\section{The conclusion}

(1) With the freezing time increased in the initial,the loss rate of the strength concrete increased. Through the analysis of the data and the prediction equation can be quantitatively obtained that with the increase of freezethaw cycles, the residual and the mass loss rate showed a significant increasing trend.

(2) Theoretical values and experimental values are consistent when compared to experimental values and theoretical values. therefor, the simulation equations can be applied in practical.

\section{Reference}

[1] Bisby Luke A, Green, Mark F. Resistance to freezing and thawing of fiber-reinforcedpolymer-co ncrete bond[J]. ACI Structural Journal,2002, 99(2): 215-223.

[2] Chengxingming. Application of gray system theory in the kinetic energy of the projectile into concrete targets in the body[D]. Mianyang:China Academy of Engineering Physics PhD thesis, 2012.

[3] Hua Xi, Wang Xiangdong, Wang Yuefeng. Research on constitutive relationship of frozon-thawe d concrete based on grey theory[J]. Journal of Disaster Prevention and Mitigation Engineering, 2013, 33(2): 185-189.

[4] Numata, Yasumasa. Study on finger pointers using gray theory[J]. Proceedings of the 3rd International Conference on Digital Society. 2009, 139-142.

[5] Kang Min Goo. Integration of hydrologic gray model with global search method for realtime flood forecasting[J]. Journal of Hydrologic Engineering, 2009, 1136-1145. 